\title{
A Rare Complication of Henoch-Schönlein Purpura: Acute Appendicitis Treated Conservatively - A Case Report and Literature Review
}

\author{
Sarmad Farook Al Hamdani ${ }^{a} \quad$ Alaa Haitham Salman ${ }^{b}$

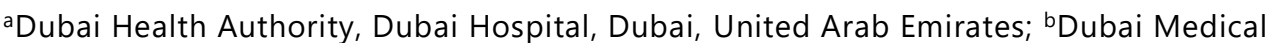 \\ College, Dubai, United Arab Emirates
}

\section{Keywords}

Henoch-Schönlein purpura $\cdot$ Vasculitis · Abdominal pain · Nephritis · Appendicitis

\begin{abstract}
Henoch-Schönlein purpura (HSP) is the most common systemic vasculitis of childhood. It is a self-limiting disorder of unknown autoimmune origin. It affects multiple organ systems including the skin, joints, the gastrointestinal (Gl) system, and the kidneys. Gl symptoms include abdominal pain, nausea, vomiting, GI bleeding and intussusception. Acute appendicitis is an extremely rare complication of HSP. When it does occur, physicians seem to rush for surgical management. However, we suggest that conservative management should be the first approach to treatment, but great caution has to be paid to the small, yet possible risk of perforation of the appendix. Here, we present a case of an 8-year-old girl, who was admitted and diagnosed with HSP. On the second day of admission, the patient developed right iliac fossa pain and tenderness, as well as seven episodes of vomiting. She was diagnosed clinically with acute appendicitis which was confirmed by abdominal ultrasound. The patient was treated successfully with conservative management without any complication.
\end{abstract}

(C) 2020 The Author(s)

Published by S. Karger AG, Basel 
Background

Henoch-Schönlein Purpura (HSP) is the most common systemic vasculitis of childhood with an incidence rate of 6.2-70.3 per 100,000 children. It shows male predominance and a peak around 4-6 years of age. The dominant clinical features of HSP are cutaneous purpura $(100 \%)$, arthritis (82\%), abdominal pain (63\%), gastrointestinal bleeding (33\%), and nephritis $(40 \%)$ [1]. Several patients present with predominantly petechial lesions, some with mainly purpuric lesions, and others with a mixture type of lesions [2]. Although uncommon, acute appendicitis can complicate HSP and was found to have occurred in 7 patients in the literature [3]. It is an extremely rare gastrointestinal complication of HSP [4]. In a study about the surgical complications in HSP, 186 patients were investigated, out of whom, only 1 developed acute appendicitis [5]. HSP is related to unspecific autoimmune origin and is usually selflimiting [6]. It demonstrates seasonal variation, implicating a role for environmental triggers and geographical variation [7]. It often follows an upper respiratory tract infection, with most cases occurring in the fall and winter. Although many cases do not have a specifically identified infectious trigger, a variety of viral and bacterial agents have been associated with HSP, group A $\beta$-hemolytic streptococcus being the most common. Pathogenesis has been linked to IgA1, a subclass of IgA, and its deposition in the glomerulus, the skin, and the blood vessels of the gastrointestinal tract [8].

HSP mostly affects skin and joints, and less often the gastrointestinal (GI) and renal systems. The usual GI symptoms of HSP include nausea, vomiting, and abdominal pain, but inflammatory manifestations like appendicitis are less likely to be present. HSP usually resolves within 4 weeks [9], and chronic complications like renal failure are not usual [6]. Diagnosing HSP is not confined to a single specific test; it is rather the co-existence of signs and symptoms that fulfill the criteria of the American College of Rheumatology. These criteria include: palpable purpura in the presence of one or more of the following: diffuse abdominal pain, any biopsy showing predominant immunoglobulin A deposition, arthritis (acute, any joint) or arthralgia, and renal involvement (any hematuria or proteinuria) [10].

Most cases are self-limiting and require only symptomatic management. For pain relief, paracetamol and a short course of non-steroidal anti-inflammatory drugs (NSAIDs), such as ibuprofen or naproxen, are useful if not otherwise contraindicated. The use of steroids (oral prednisolone or intravenous methylprednisolone) has been shown to reduce the duration of abdominal and joint pain, but does not affect the rate of long-term renal complications of HSP. Once symptoms have resolved, an appropriate weaning regimen should be used [11].

While abdominal pain is a common occurrence in HSP, it should not be disregarded, but rather carefully assessed to exclude cases that would need surgical intervention such as intussusception, perforation, necrosis, and massive gastrointestinal bleeding. There is not enough evidence to suggest the need for urgent appendectomy in cases of suspected appendicitis occurring with HSP, rather a more conservative approach may be undertaken, with careful observation and assessment by pediatric surgeons to prevent perforation of the appendix when suspected. Clinical surveillance along with serial ultrasonographic pictures are needed to detect conditions when surgery would be necessary in patients with HSP.

\section{Case Presentation}

An 8-year-old previously healthy girl presented to the emergency department with a 2day history of purpuric rash over the trunk, upper and lower limbs, a 2-day history of bilateral 
ankle swelling with increasing tenderness, and a 1-day history of vomiting. She had vomited thrice, including one episode with streaks of blood. She had normal bowel movements with no blood in stool. The patient has a first-degree family history of systemic lupus erythematosus (SLE). On examination, the patient was conscious and alert. Vital signs showed a pulse rate of $138 \mathrm{bpm}$, a blood pressure of $111 / 67 \mathrm{~mm} \mathrm{Hg}$, and a temperature of $37.2^{\circ} \mathrm{C}$.

On the day of admission, laboratory investigation showed a WBC count of $14 \times 10^{3} / \mu \mathrm{L}$ and a platelet count of $449 \times 10^{3} / \mu \mathrm{L}$. Urine routine showed protein $1+$, with $20-25 \mathrm{RBCs} / \mathrm{HPF}$ and $10 \mathrm{WBCs} / \mathrm{HPF}$. Her serum CRP was $40 \mathrm{mg} / \mathrm{dL}$ and her ESR was 35. Serum electrolytes, liver enzymes, and blood coagulation tests were unremarkable. Fecal occult blood test was positive.

In view of her family history of SLE, we checked the patient's serum for anti-nuclear antibody (ANA) and anti-dsDNA which came out negative. Her C3 and C4 levels were normal (1.32 and 0.27, respectively). Her serum IgA was $1.62 \mathrm{~g} / \mathrm{L}$ (normal range: 0.33-2.00 g/L). These results excluded a diagnosis of collagen vascular disease, and thus she was diagnosed with HSP and was started on intravenous (i.v.) fluids, ibuprofen, esomeprazole, with 2 hourly blood pressure surveillance and once daily urine routine check. On the next day, she complained of right iliac fossa abdominal pain and tenderness, the suspicion of appendicitis or intussusception was made clinically. She was kept NPO. An abdominal ultrasound was performed (Fig. 1), which showed a picture suggestive of early acute appendicitis. The ultrasound showed a tubular non-compressible peristaltic structure (the appendix) in the right lower quadrant with a small amount of fluid adjacent to it. The appendix measured $5.5 \mathrm{~mm}$ and was not significantly thickened, suggesting early inflammation. Periappendiceal echogenic mesentery was suggestive of acute inflammatory changes.

A pediatric surgeon was consulted, who confirmed the diagnosis of appendicitis and decided on close monitoring and conservative treatment with consideration of surgical exploration if the patient's condition deteriorated. The patient was started on i.v. methylprednisolone, $1 \mathrm{mg} / \mathrm{kg} 6$ hourly in view of significant abdominal pain and was kept NPO with maintenance intravenous fluids and pain medication.

That evening, the patient vomited 5 times, yellowish brown vomitus with streaks of blood in the initial episodes, and new rashes appeared over her upper limbs and cheeks. However, her abdominal pain improved and was controlled with the pain medication.

On the next day, she had two blood pressure readings that were more than the $95^{\text {th }}$ percentile for her age $(132 / 85,130 / 75 \mathrm{~mm} \mathrm{Hg})$, with a urinalysis showing protein $3+$ with RBCs and WBCs, and a protein to creatinine ratio of 0.6 . The patient was referred to the pediatric nephrology team who advised to start angiotensin-converting enzyme Inhibitors (ACEIs), and investigate further for renal vasculitis.

On day 3 of admission, her abdominal pain settled and she was started on a soft diet, which was well tolerated, and she passed normal stool. The pediatric surgeon confirmed resolution of the acute appendicitis. On day 4 of admission, the patient complained of mild abdominal pain again which was periumbilical, and vomited twice a greenish vomitus; therefore, an abdominal ultrasound was repeated, (Fig. 2), which showed persisting small amounts of free fluid in the right lower abdomen on graded compression technique. The appendix could not be visualized due to bowel gases. However, there was no obvious bowel mass to the visualized extent. The liver, spleen, pancreas, gallbladder, and kidneys were unremarkable.

The pediatric surgeons were contacted for the findings. They asked for an urgent abdominal X-ray (which appeared normal, see Fig. 3) and advised to keep the patient NPO for 24 $\mathrm{h}$, then to gradually introduce diet as sips of water, to give a glycerin suppository. The next day, her abdominal pain improved and she had no more episodes of vomiting. Therefore, the 
patient was settled on conservative management and had no further complaints of abdominal pain or vomiting, despite not having received antibiotics or any treatment other than pain control. On repeat blood analysis, her WBC count was $13 \times 10^{3} / \mu \mathrm{L}$, CRP was $20 \mathrm{mg} / \mathrm{dL}$, and ESR was not repeated. The purpuric rash was still present, despite the improvement in her abdominal complaints.

However, the patient continued having nephrotic range proteinuria along with hematuria (dipstick urinalysis showed 3+ proteinuria with 5-10 RBCs and WBCs per HPF); so, three renal needle biopsies were taken, then she was given three doses of pulse methylprednisolone.

After the biopsy, the patient was discharged with the following medications: prednisolone, esomeprazole, lisinopril, and Cellcept.

The biopsy (Fig. 4) showed immunofluorescence findings of IgA dominant deposits. A total of 24 glomeruli were sampled under light microscopy, none of which were sclerotic. While the majority of the glomeruli appeared hypercellular, seven were involved by active crescent formation, and six showed endocapillary proliferation without crescent formation. Although no chronicity was spotted, the activity appeared to be severe. The glomeruli displayed granular mesangial and segmental capillary wall staining that was 3+ for IgA, 2+ for IgG, kappa and lambda and trace to 1+ for C3. Staining for IgM and C1q was negative. A final diagnosis of diffuse proliferative glomerulonephritis was made.

Sixteen days after the biopsy, the patient presented with a history of fever with chills for a few hours, and mild burning micturition and hematuria for the past 3 days. She was seen in a private clinic where urine routine and culture were collected. Urine routine showed pale red, turbid urine with protein 1+, 30-35 WBCs/HPF, and numerous RBCs. Urine culture grew E. coli with $>100,000 \mathrm{CFU} / \mathrm{mL}$. Therefore, she was diagnosed with a urinary tract infection (UTI) caused by E. coli, sensitive to nitrofurantoin. She was admitted and given p.o. cefuroxime to which she responded clinically well and after being afebrile for more than $48 \mathrm{~h}$, she was discharged on nitrofurantoin for 10 days. Repeat urinalysis 12 days later showed clear urine with no proteinuria, 0-5 WBCS/HPF, and 15-20 RBCs/HPF. Repeat urine culture was negative.

The follow-up plan was to taper down prednisolone within 4 weeks. If no proteinuria was found, then we would taper mycophenolic acid (Cellcept) to $500 \mathrm{mg}$ b.i.d. with continuation of other prescribed medications.

\section{Discussion}

The main aim of our case report was to highlight that conservative management with close observation is possible to prevent an unnecessary appendectomy in a child with HSP. Our 8-year-old patient who developed right iliac fossa pain on day 2 of admission, followed by several episodes of green vomitus, was diagnosed with acute appendicitis and showed subsequent improvement on supportive management. However, meticulous clinical judgment, along with surgical consultation and close follow-up are needed to prevent complications such as perforation of the appendix.

An important point is that vasculitic involvement of the ileum or ascending colon may produce signs that mimic acute appendicitis and lead to an unnecessary appendectomy [12, 13]. This misdiagnosis has been reported to occur as often as $5-7 \%$ in two series $[13,14]$.

However, the ultrasonographic and X-ray images that we obtained in our case were highly suggestive of appendicitis, ruling out the possibility of this being a misdiagnosis of vasculitis of the ileum or ascending colon. 
Since abdominal manifestations may precede the appearance of the rash in HSP, it is important that we include HSP in the differential diagnosis of abdominal pain so that we avoid unnecessary surgical procedures [15]. One report described a 16-year-old boy who underwent surgery due to abdominal pain, mimicking appendicitis. Intraoperatively, however, the appendix and ileum showed macroscopic signs of vasculitis, without inflammation. The classical purpuric rash and joint pain of HSP appeared 4 days postoperatively, confirming the diagnosis of HSP [16].

It is through the use of abdominal ultrasonography and X-rays, when indicated, along with a good physical examination, that surgical complications of intussusception and appendicitis in HSP are suspected and their progression followed up, therefore avoiding unnecessary laparotomies and laparoscopies [17].

On the other hand, as important as it is to avoid unnecessary laparotomies, it is also crucial to suspect and intervene early when surgical emergencies are assumed, including perforated appendix, intestinal obstruction, and hemorrhage, though perforated appendix may still be managed nonoperatively, with antibiotics [18].

After reviewing the literature, we found a case report describing a 7-year-old boy who developed perforation of the appendix on day 5 of admission to the hospital and underwent an appendectomy. The perforated appendix was confirmed by ultrasonography prior to surgery [19]. Another case described a 6-year-old who also developed perforation of the appendix and a periappendiceal abscess, and the diagnosis of HSP was only made postoperatively as the rash appeared on the fifth postoperative day [20]. In addition, 3 HSP cases with appendicitis were reported, where appendectomy was performed, and in all of them, the operation was done prior to the appearance of the rash. One of these cases was found to have a perforated appendix [21].

We believe that our case is the first reported case of appendicitis occurring with HSP that was successfully managed conservatively. It is now known that appendicitis can be managed with antibiotics in the first $12 \mathrm{~h}$ of symptom onset, ideally within the first $6 \mathrm{~h}$ [22]. Our patient, on the other hand was not given antibiotics, but still responded and improved without any intervention.

Although some may point to the possibility of steroids masking the diagnosis of appendicitis, in our case, steroids, which were given for the HSP nephritis, were only started following the diagnosis of appendicitis and so could not have masked the signs and symptoms of appendicitis. In addition, it is mostly patients who take steroids chronically that seem to get a "silent appendicitis" [23].

\section{Conclusion}

HSP is a multisystem self-limiting autoimmune vascular disorder. Our patient developed two of the several complications that can occur with HSP, namely renal (diffuse proliferative glomerulonephritis) and abdominal (appendicitis). The acute appendicitis resolved with supportive management and her vomiting and abdominal pain gradually subsided. However, her renal complications persisted for some time, and she was followed up in the pediatric nephrology clinic for 4 months from the initial diagnosis. During that time, she was continued on prednisolone (which was gradually tapered down), nexium, lisinopril and CellCept.

Despite some belief that a laparotomy or a laparoscopy should always be done if there are concerns that a patient with HSP has developed acute appendicitis, we believe that appendicitis in association with HSP can be treated conservatively, omitting the many complications 
that can occur during surgery. We hope that this case report promotes further research into this topic to help physicians decide on the most appropriate management of appendicitis in patients with HSP, avoiding unneeded surgical interventions, yet recognizing conditions when immediate surgery is essential.

\section{Statement of Ethics}

The study protocol was approved by the Institute's Committee on Human Research. A written informed consent was obtained from the family to publish their case including publication of the images of investigations. There are no patient's identifiers in the case report which may link the patient to the report.

\section{Disclosure Statement}

The authors have no conflicts of interest to declare.

\section{Funding Sources}

No funding was received.

\section{Author Contributions}

Both authors contributed equally to this report.

\section{References}

1 Saulsbury FT. Henoch-Schönlein purpura in children. Report of 100 patients and review of the literature. Medicine (Baltimore). 1999 Nov;78(6):395-409.

2 Leung AK, Chan KW. Evaluating the child with purpura. Am Fam Physician. 2001 Aug;64(3):419-28.

3 De Castro SM, Joosse P, Unlü C, Steller EP. Henoch-Schonlein Disease Localized in the Appendix. Indian J Pediatr. 2013 Dec;80(12)1047-9.

4 Humes DJ, Simpson J. Acute appendicitis. BMJ. 2006 Sep;333(7567):530-4.

5 Mir E. Surgical complications in Henoch-Schönlein Purpura in childhood. Z Kinderchir. 1988 Dec;43(6):3913.

6 Marcdante K. Nelson essentials of pediatrics, international edition. Elsevier Health Science; 2018.

7 Oni L, Sampath S. Childhood IgA Vasculitis (Henoch Schonlein Purpura)-Advances and Knowledge Gaps. Front Pediatr. 2019 Jun;7(7):257.

8 Reid-Adam J. Henoch-Schonlein purpura. Pediatr Rev. 2014 Oct;35(10):447-9.

9 Saulsbury FT. Henoch-Schönlein purpura. Curr Opin Rheumatol. 2001 Jan;13(1):35-40.

10 Reamy BV, Williams PM, Lindsay TJ. Henoch-Schonlein Purpura. Am Fam Physician. 2009 Oct;80(7):697704.

11 Henoch-schonlein purpura. The Royal Children's Hospital Melbourne. Clinical Practice Guidelines. 2016 Nov.

12 Feldt RH, Stickler GB. The gastrointestinal manifestations of anaphylactoid purpura in children. Proc Staff Meet Mayo Clin. 1962 Aug;37(37):465-73.

13 Martinez-Frontanilla LA, Haase GM, Ernster JA, Bailey WC. Surgical complications in Henoch-Schönlein purpura. J Pediatr Surg. 1984 Aug;19(4):434-6.

14 Katz S, Borst M, Seekri I, Grosfeld JL. Surgical evaluation of Henoch-Schönlein purpura. Experience with 110 children. Arch Surg. 1991 Jul;126(7):849-53. 
Al Hamdani and Salman: A Rare Complication of HSP: Acute Appendicitis Treated Conservatively

15 Fan Z, Tian X, Pan J, Li Y, Zhang Y, Jing H. Terminal Ileitis Induced by Henoch-Schonlein Purpura That Presented as Acute Appendicitis - a case report. Medicine (Baltimore). 2015 Feb;94(5):e492.

16 de Castro SM, Joosse P, Unlü C, Steller EP. Henoch-Schönlein disease localized in the appendix. Indian J Pediatr. 2013 Dec;80(12):1047-9.

17 Connolly B, O'Halpin D. Sonographic evaluation of the abdomen in Henoch-Schonlein purpura. Clin Radiol. 1994 May;49(5):320-3.

18 Howell EC, Dubina ED, Lee SL. Perforation risk in pediatric appendicitis: assessment and management. Pediatric Health Med Ther. 2018 Oct; 9:135-45.

19 Kim CJ, Chung HY, Kim SY, Kim YO, Ryu SY, Kim JC, et al. Acute appendicitis in Henoch-Schönlein purpura: a case report. J Korean Med Sci. 2005 Oct;20(5):899-900.

20 Ohno Y, Ohgami H. Simultaneous occurrence of Henoch-Schönlein purpura and acute appendicitis. Pediatr Surg Int. 1995;10(8):563-4.

21 Bryce A. Binstadt, Eric W. Fleegler. Perforated appendicitis in a child with Henoch-Schönlein purpura. J Pediatr Surg. 2004;10:002.

22 Coccolini F, Fugazzola P, Sartelli M, Ciccutin E, Sibilla MG, et al. Conservative treatment of acute appendicitis. Acta Biomed. 2018 Dec;89(9):119-34.

23 Anshu Fl, Naids JM, Thakur K. Silent Appendicitis in an Immunocompromised Patient: A Diagnostic Dilemma. AJG. 2017 Oct; 112:S1317-S1318

The work was conducted at the Dubai Hospital/Dubai Health Authority Pediatric Department, Dubai, United Arab Emirates.

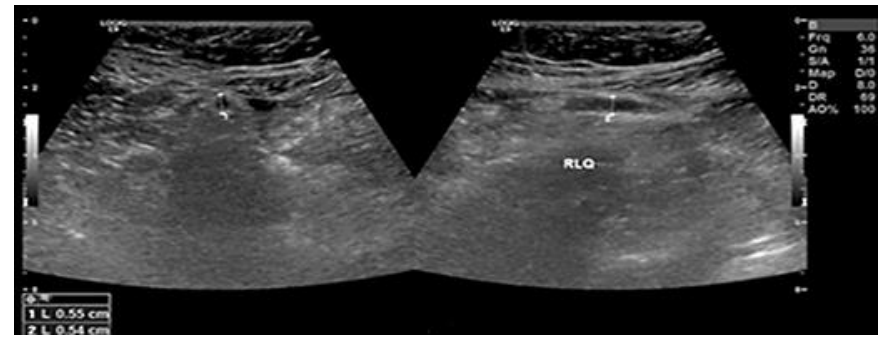

Fig. 1. The first abdominal ultrasound showing signs of early acute appendicitis.

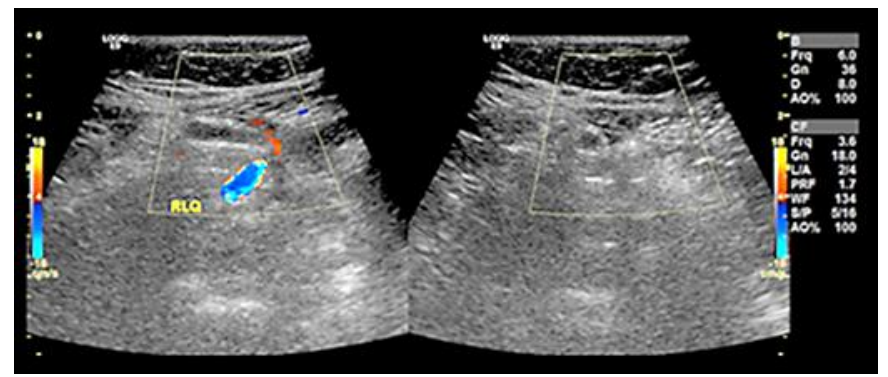

Fig. 2. The second abdominal ultrasound showing persisting small amounts of free fluid in the right lower abdomen. 


\section{Case Reports in Acute Medicine}

Case Rep Acute Med 2020;3:17-24

DOI: $10.1159 / 000507429$ (c) 2020 The Author(s).
www.karger.com/cra

Al Hamdani and Salman: A Rare Complication of HSP: Acute Appendicitis Treated Conservatively
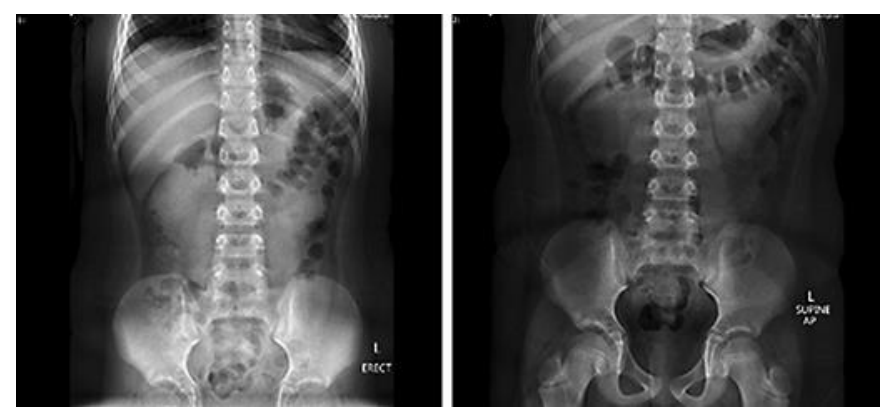

Fig. 3. Normal abdominal X-ray.

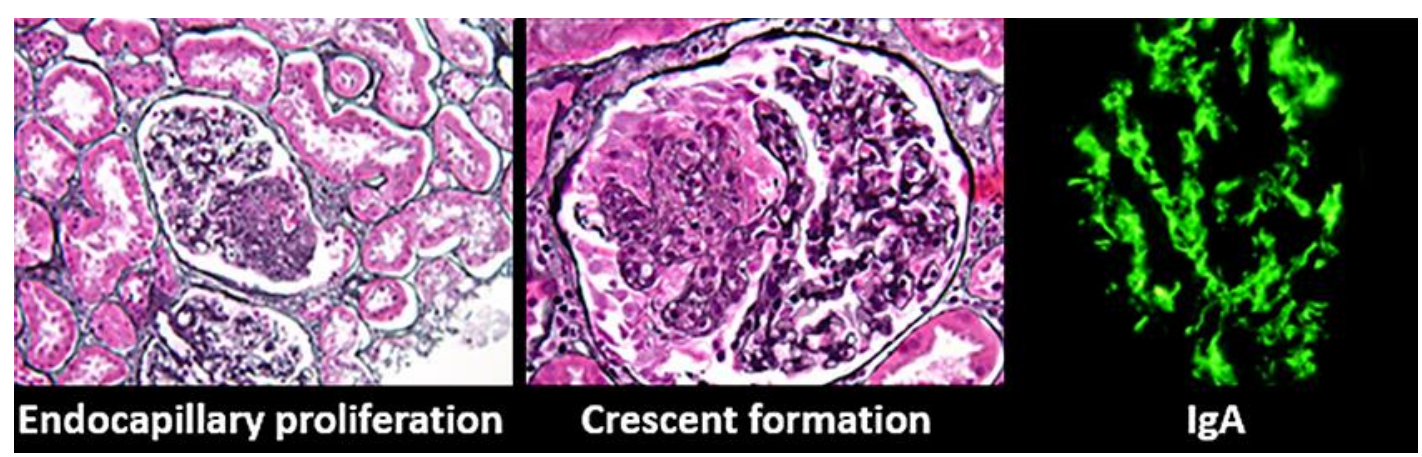

Fig. 4. Renal biopsy showing immunofluorescence findings of IgA-dominant deposits. 